\title{
Temporal Changes in Bystander Cardiopulmonary Resuscitation Rates in \\ England
}

Terry Brown ${ }^{1}$, Claire Hawkes ${ }^{1}$, Scott Booth ${ }^{1}$, Rachael Fothergill ${ }^{2}$, Sara Black ${ }^{3}$, Anna Bichman ${ }^{4}$, Helen Pocock ${ }^{5}$, Jasmeet Soar ${ }^{6}$, Julian Mark ${ }^{7}$, Jonathan Benger ${ }^{8}$, Gavin Perkins ${ }^{1}$, OHCAO collaborators

\section{Purpose of the Study:}

Bystander CPR (BCPR) more than doubles the chances of survival from out-of-hospital cardiac arrest (OHCA). In the UK BCPR rates are variable and lower than those of other countries. This is the first study to investigate the trends in BCPR rates in England using data collected by the OHCAO registry.

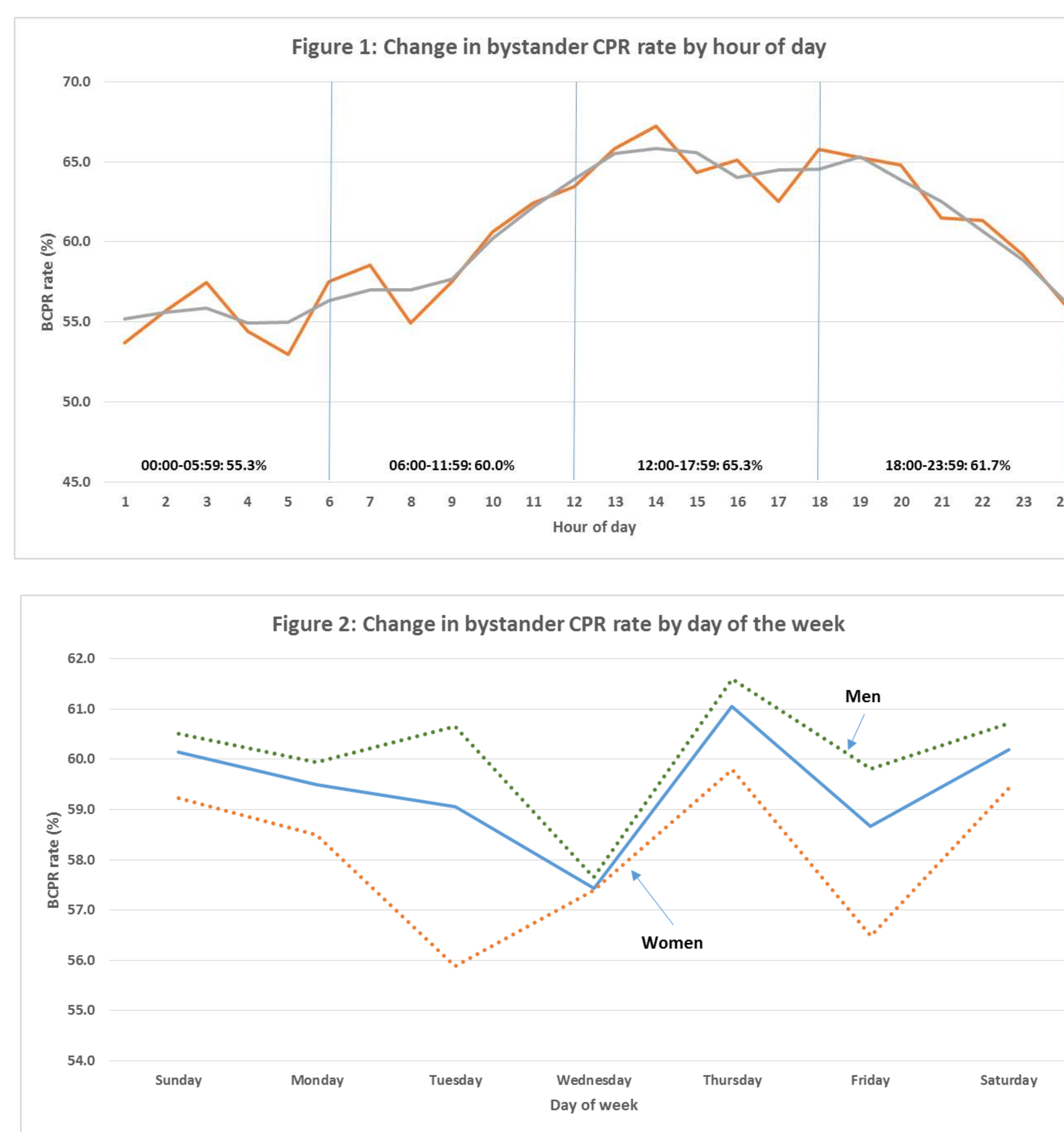

Results:

The odds (OR) of having an OHCA during the day was more than double that in the evening/night $(\mathrm{OR}=2.28)$. The highest proportion of OHCAs occurred on a Monday (Sunday for women $\&<65 \mathrm{y}$ ). December had the greatest number of OHCAs, but the OR of having an OHCA in winter was 0.83 compared to autumn.

$B C P R$ rate increased during the morning to about lunchtime, plateaued until about 19:00h then declined (Figure 1). The OR of receiving BCPR was significantly higher during the day compared to the evening/night (OR=1.14; 95\% $\mathrm{Cl}=1.08-1.19)$.

$B C P R$ rates were greater at the weekend than mid-week ( $O R=1.04$; $95 \% \mathrm{Cl}=0.99-1.10$ ) (Figure 2). BCPR rate was $61.9 \%$ in OHCAs that occurred during regular working hours (58.0\% out of hours). BCPR rate showed an annual pattern, peaking in Oct/Nov \& minimum around Apr/Jun. OR of receiving BCPR during the autumn/winter were significantly higher compared to spring/summer (OR=1.07; 95\%Cl=1.02-1.12) (Figure 3). BCPR rates have increased significantly from $51 \%$ in April 2013 to $66 \%$ in December $2015(p<0.05)$ (Figure 4); the increase being observed for both men and women, and most age groups. However, the trend varied between ambulance services.

Joinpoint regression analysis showed there was significant variation during this time; an increase in February 2014, dips in May 2014 and March 2015, and an increase around June 2015 (Figure 4).

\section{WARWICK}

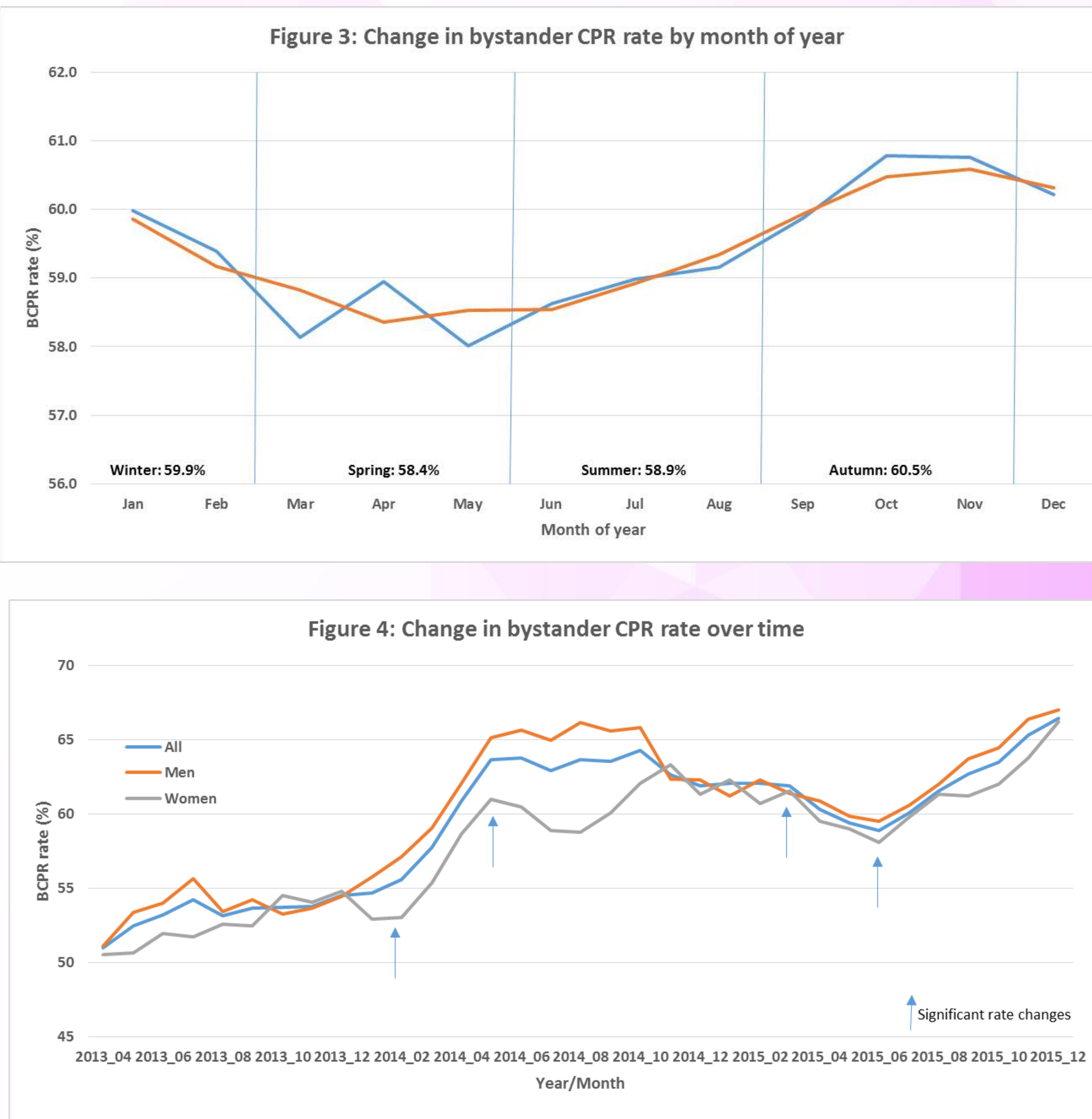

Conclusions:

We have shown for the first time in England significant temporal variability in $\mathrm{OHCA}$ and BCPR incidence. The trend of increasing BCPR is encouraging and likely to be associated with national and local initiatives to improve BCPR rates. However, there is still room for improvement in specific areas of the country, and a need for continued awareness programmes. 